Rapid Communications

\title{
Fluorescence Enhancement Effect by Metal Nanoparticles-immobilized Microplate
}

\author{
Shuyi Sun, * Kyohei Matsui, ${ }^{*}$ So Tanabe, * Dung Nguyen, ${ }^{*}$ Takamasa Kinoshita,* \\ Yojiro YAMAMOTo, ${ }^{* * *}$ and Hiroshi SHIIGI* ${ }^{* \dagger}$ \\ *Department of Applied Chemistry, Osaka Prefecture University, 1-2 Gakuen, Naka, Sakai, Osaka 599-8570, \\ Japan \\ **GreenChem. Inc., 19-19 Tsuruta, Nishi, Sakai, Osaka 593-8323, Japan
}

\begin{abstract}
In this reported work, we achieved high-throughput, highly sensitive fluorescent analysis using an enzyme-linked immunosorbent assay (ELISA) that employed a metallic nanoparticle (NP)-immobilized 96-well plate. The immobilization of metallic NPs on a 96-well plate effectively amplified fluorescent signals of the assay. The silver (Ag) NP-immobilized plate showed the best fluorescent enhancement effect of all plates immobilized by metal NPs. Our results demonstrate the potential of applying Ag NPs to enhance the efficiency of direct and indirect ELISA by the labeling of targets.
\end{abstract}

Keywords Microplate, silver nanoparticle, fluorescence enhancement, ELISA

(Received June 11, 2020; Accepted June 22, 2020; Advance Publication Released Online by J-STAGE June 26, 2020)

In our previous work, we demonstrated a fluorescence enhancement effect of gold nanoparticles (NPs). ${ }^{1}$ The fluorescence intensity of dyes was enhanced or quenched, depending on their distance from and orientation relative to the surface of metallic NPs, ${ }^{2}$ which was caused by interactions between the dyes and the localized surface plasmon resonance (LSPR) coupling generated from metallic NPs. ${ }^{3-9}$ Therefore, this effect might enable us to utilize metallic NPs on microplates to amplify the fluorescence signal. In this study, various kinds of metallic NPs were immobilized inside the wells of a microplate. A sodium hydroxide aqueous solution $(1.0 \mathrm{M}, 0.74 \mathrm{~mL})$ was added to $100 \mathrm{~mL}$ of an aqueous solution containing $0.25 \mathrm{mM}$ silver nitrate and $0.16 \mathrm{mM}$ EDTA-2Na under vigorous stirring at $373 \mathrm{~K}$. A yellowish Ag NP dispersion (0.0027 wt\%) was obtained after stirring for $5 \mathrm{~min}$. The $\mathrm{pH}$ of the dispersion was adjusted to 4.0 by the addition of hydrochloric acid $(1.0 \mathrm{M}$, $0.65 \mathrm{~mL})$ under vigorous stirring. The dispersion $(0.45 \mathrm{~mL})$ was added to a 96-well plate and incubated for $12 \mathrm{~h} .{ }^{10}$ After the dispersion was removed, the amino-functionalized 96-well plate (Sumitomo Bakelite Co., Japan) was dried at $333 \mathrm{~K}$ for $1 \mathrm{~h}$. The preparation of the 96-well plates immobilizing other metal NPs is described in Supporting Information.

The mean diameter of the synthesized Ag NPs was $32.4 \pm$ $5.1 \mathrm{~nm}$. The absorption spectra of the Ag NPs dispersion in the well showed one peak at $406 \mathrm{~nm}$, attributable to Ag NPs in the dispersed state (Fig. 1A). ${ }^{11}$ The Ag NPs maintained the dispersion state for a month, since they repelled each other in an aqueous solution due to their negative zeta potential $(-35.4 \mathrm{mV})$. It was presumed that negatively charged EDTA molecules surrounded the Ag NPs since four carboxylic groups of EDTA were dissociated and two tertiary amine groups were protonated at $\mathrm{pH}$ 4.0. The absorbance of the dispersion decreased notably

† To whom correspondence should be addressed.

E-mail: shii@chem.osakafu-u.ac.jp without a peak shift after incubation of the dispersion in the well for $12 \mathrm{~h}$. On the other hand, the inside of the well was uniformly stained yellow, indicating that the Ag NPs were uniformly immobilized inside the well (Fig. 1B). The yellow color on the well became deeper upon increasing the concentration of the incubated Ag NP dispersion. The wells in the 96-well plate were positively charged at $\mathrm{pH} 4.0$ owing to protonation of the functionalized amine groups that had already modified the surface of each well. Therefore, the Ag NPs were simply immobilized on the wells through electrostatic interactions.

The amount of immobilized Ag NPs on the 96-well plate, determined by subtracting the absorbance of the Ag NP dispersion at $406 \mathrm{~nm}$ removed after incubation from that of the prepared Ag NP dispersion before incubation, showed a linear relationship with the concentration of the incubated dispersion $\left(R^{2}=0.9949\right.$, Fig. 2A). When the concentration was the highest $(0.0027 \mathrm{wt} \%), 9.6 \mu \mathrm{g}\left(4.2 \mu \mathrm{g} \mathrm{cm}^{-2}\right)$ of Ag NPs was immobilized on the well. The fluorescence intensity of each well at $535 \mathrm{~nm}$ was measured with a plate reader (Ex. $485 \mathrm{~nm}$ ) after naturally drying the fluorescein aqueous solution $(0.10 \mathrm{mM}, 40 \mu \mathrm{L})$ in a dark room at $298 \mathrm{~K}$ for $12 \mathrm{~h}$. Despite an immobilized amount of fluorescein, the fluorescence intensity increased linearly upon increasing the amount of Ag NPs. This indicates that the Ag NPs on the well enhanced the fluorescence of the fluorescein molecules (Fig. 2B). Moreover, the fluorescence enhancement was accelerated when the Ag NP content was above $4.8 \mu \mathrm{g}$ $\left(2.1 \mu \mathrm{g} \mathrm{cm}^{-2}\right)$. A stronger electric field was known to be induced among the particles when LSPR coupling occurred, resulting in a more efficient fluorescence enhancement. ${ }^{3,4}$ Hence, as the amount of Ag NPs increased, Ag NPs were brought into proximity to each other, and formed a nanometer-sized gap, which may be favorable for the generation of the LSPR coupling, and which would lead to an accelerated fluorescence enhancement. ${ }^{1}$ 


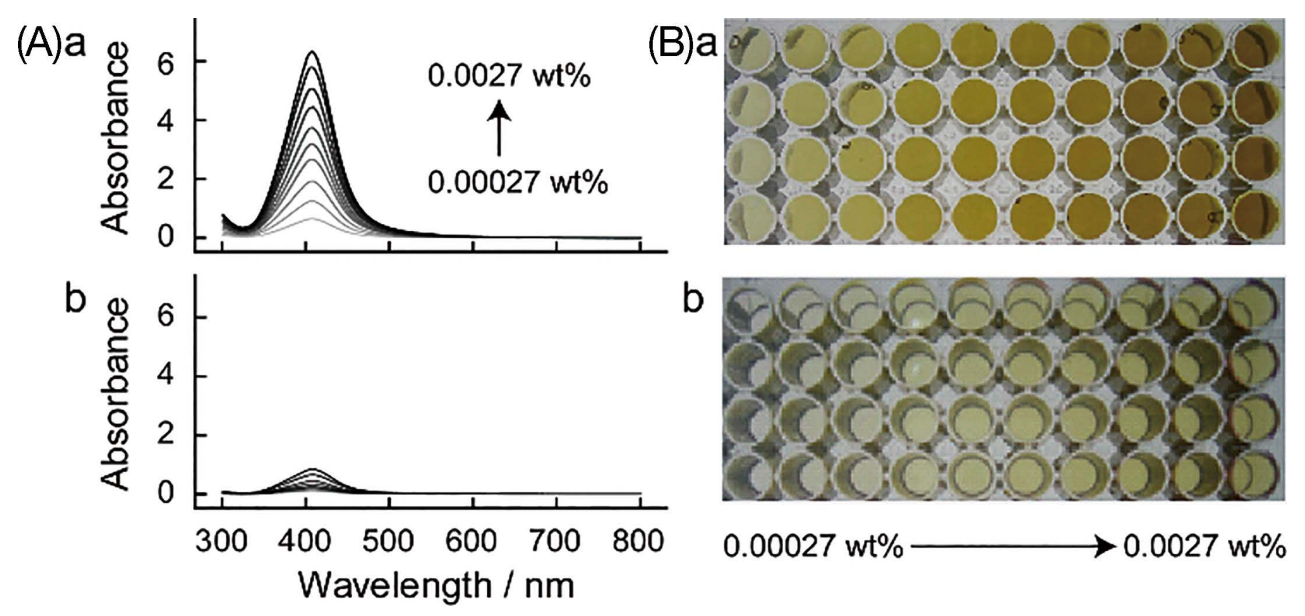

Fig. 1 (A) Absorption spectra and (B) digital photographs of (a) EDTA-covered Ag NP dispersion in the well and (b) the Ag NP-immobilized well after incubating for $12 \mathrm{~h}$.

(A)

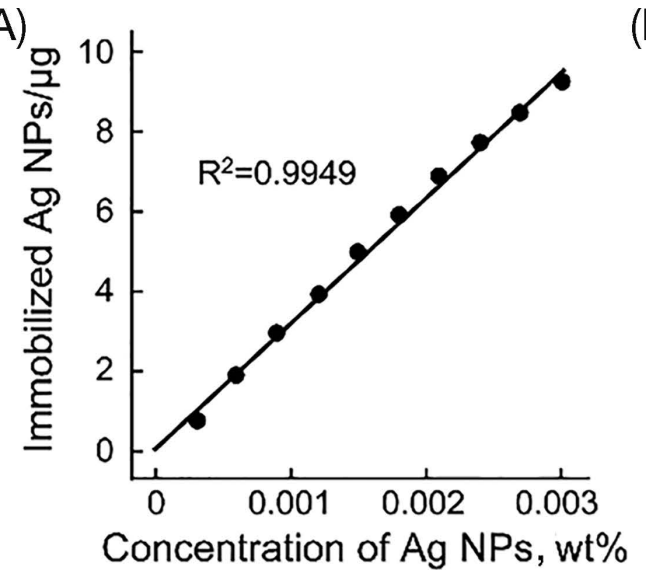

(B)

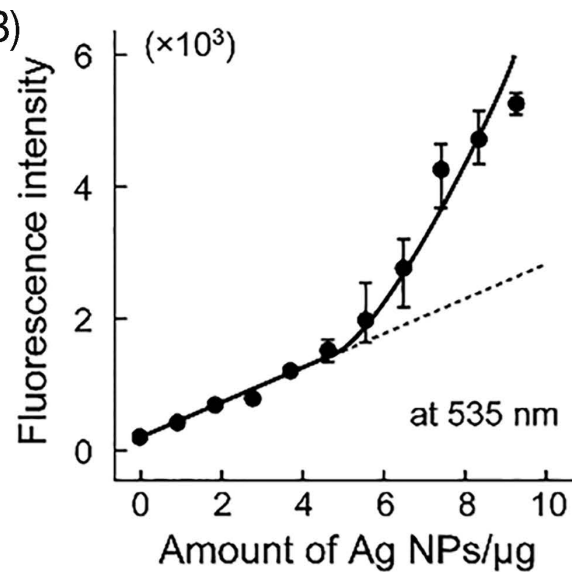

Fig. 2 (A) Relationship between the amount of Ag NPs immobilized on the well and the concentration of Ag NP dispersion. (B) Dependence of the fluorescence intensity of fluorescein at $535 \mathrm{~nm}$ on the amount of immobilized Ag NPs $(N=10)$.

(A) $\mathrm{Ag} / 30 \mathrm{~nm}$
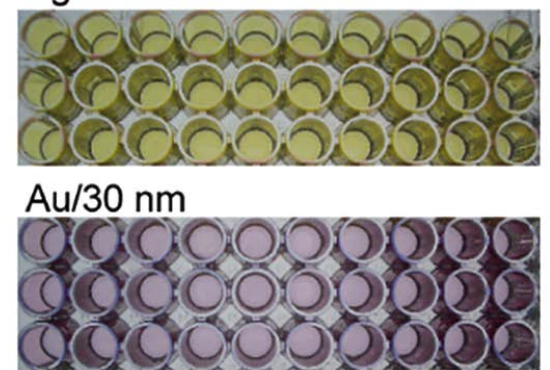

$\mathrm{Pd} / 40 \mathrm{~nm}$

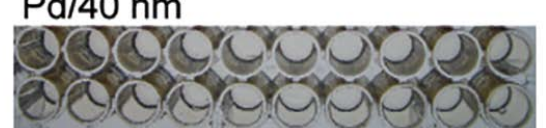

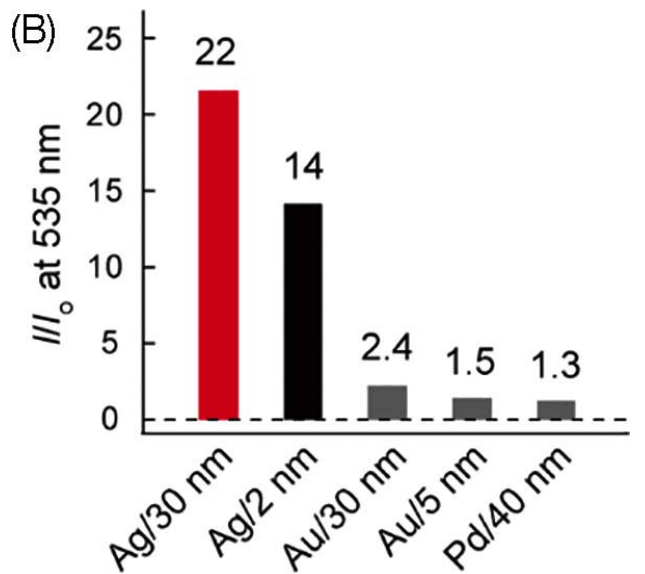

Fig. 3 (A) Digital photographs of 96-well plate immobilized Ag, Au, and Pd NPs. (B) Fluorescence enhancement efficiency $\left(I / I_{\mathrm{o}}\right)$ of $\mathrm{Ag}$, Au, and Pd NP-immobilized wells. $I$ and $I_{\mathrm{o}}$ represent the fluorescence intensity of the fluorescein molecule in a NP-immobilized well and the unmodified well, respectively. 
We applied the one-step immobilization method for gold (Au) and palladium (Pd) NPs (see Supporting Information). Both kinds of NPs showed a negative zeta potential (Au NPs: $-31.2 \mathrm{mV}$, Pd NPs: $-34.4 \mathrm{mV}$ ) owing to negatively charged citrate molecules surrounding the NPs, since two carboxylic groups of citrate were dissociated at $\mathrm{pH} 4.0$. They were uniformly immobilized on the well owing to electrostatic interactions. The insides of the wells were stained reddish purple and black, respectively (Fig. 3). This was due to the LSPR inherent in metal NPs. LSPR is strongly dependent not only on the metal species, but also on the size, shape, aggregation state, and local environment. ${ }^{1,8,9}$ Given these equal conditions on the wells, metal species-based LSPRs appeared at different wavelengths, and thus were deeply involved in the fluorescence enhancement. The fluorescence intensity of each well $(I)$ was measured after incubation of the fluorescein aqueous solution $(0.10 \mathrm{mM}, 40 \mu \mathrm{L})$ for $12 \mathrm{~h}$, and removal of the supernatant. The fluorescence enhancement was confirmed for the Au NP$\left(I / I_{\mathrm{o}}=2.4\right)$ and Pd NP-immobilized well $\left(I / I_{\mathrm{o}}=1.3\right)$ compared with that for the unmodified well $\left(I_{0}=\sim 213\right)$. In addition, it was found that immobilized NPs of the same metal that had a larger diameter led to greater fluorescence enhancement. ${ }^{1,2}$ We found that the 96-well plate immobilizing $30 \mathrm{~nm} \mathrm{Ag} \mathrm{NPs}$ $\left(4.2 \mu \mathrm{g} \mathrm{cm}^{-2}\right)$ resulted in a largest fluorescence enhancement for fluorescein $\left(I / I_{0}=22\right)$.

The Ag NP-immobilized 96-well plates have been established to enhance the fluorescent signal. Applying this plate to an ELISA is expected to improve the sensitivity of the assay. Furthermore, the labeling of various target species based on the specific binding of antibodies, aptamers, and molecularly imprinted polymers allows for a high throughput and sensitive detection using the proposed procedure.

\section{Acknowledgements}

This study was mainly supported by a JST A-STEP Grant (Number MP27115662969), Japan. We also acknowledge financial support provided by a JST START Grant (Number
JPMJST1916) and the Ministry of Agriculture, Forestry, and Fisheries through a Science and Technology Research Promotion Program for the agriculture, forestry, fisheries, and food industries.

\section{Supporting Information}

Further information is available regarding the experimental details. This material is available free of charge on the Web at http://www.jsac.or.jp/analsci/.

\section{References}

1. T. Kinoshita, D. Q. Nguyen, T. Nishino, H. Nakao, H. Shiigi, and T. Nagaoka, Anal. Sci., 2015, 31, 487.

2. S. Vukovic, S. Corni, and B. Mennucci, J. Phys. Chem. C, 2009, 113, 121.

3. F. Cannone, G. Chirico, A. R. Bizzarri, and S. Cannistraro, J. Phys. Chem. B, 2006, 110, 16491.

4. H. Su, Y. Zhong, T. Ming, J. Wang, and K. S. Wong, J. Phys. Chem. C, 2012, 116, 9259.

5. H. Shiigi, M. Fukuda, T. Tono, K. Takada, T. Okada, L. Q. Dung, Y. Hatsuoka, T. Kinoshita, M. Takai, S. Tokonami, H. Nakao, T. Nishino, Y. Yamamoto, and T. Nagaoka, Chem. Commun., 2014, 50, 6252.

6. T. Kinoshita, K. Kiso, D. Q. Le, H. Shiigi, and T. Nagaoka, Anal. Sci., 2016, 32, 301.

7. T. Kinoshita, D. Q. Nguyen, D. Q. Le, K. Ishiki, H. Shiigi, and T. Nagaoka, Anal. Chem., 2017, 89, 4680.

8. H. Shiigi, S. Kimura, T. Fujita, and T. Nagaoka, Anal. Sci., 2015, 31, 577.

9. H. Shiigi, T. Fujita, X. Shan, M. Terabe, A. Mihashi, Y. Yamamoto, and T. Nagaoka, Anal. Sci., 2016, 32, 281.

10. X. Shan, T. Yamauchi, Y. Yamamoto, H. Shiigi, and T. Nagaoka, Analyst, 2018, 143, 1568.

11. S. Choi, S. Park, and J. Yu, Chem. Commun., 2014, 50 , 15098. 